\title{
El problema de la corrección en el Arbitraje en Equidad: una propuesta de modelo prescriptivo*
}

Fernando Bajaña Tovar**

Recibido/Received: 30/06/2019

Aceptado/Accepted: 01/07/2019

Sumario: 1. Introducción. 2. Presupuestos conceptuales. 2.1 Un modelo prescriptivo. 2.2 La idea de corrección y su adopción en el presente modelo. 2.3 El objeto del juicio de corrección: La decisión en Equidad. 3. De la concepción formal a la concepción material del razonamiento jurídico. 3.1 Razones sustantivas. 3.2 El juicio de corrección: las razones y la decisión en Equidad. 3.2.1 La decisión en Equidad, una forma de regulación ex post de los costos de transacción. 4. Conclusión.

RESUMEN: El régimen arbitral ecuatoriano establece dos posibilidades para el contenido motivo de sus fallos: (i) una fundamentación en derecho, sustentada en premisas legislativas, jurisprudenciales y doctrinales; y (ii) una argumentación inspirada en equidad, donde se conjugan estándares generales como el "leal saber y entender" y la "sana crítica". Es este último criterio, de aparentes inspiraciones éticas y filosóficas, el que graba dentro el arbitraje una mayor relevancia normativa, en la medida de que la ley arbitral

* El presente artículo es una adaptación del Trabajo de Titulación presentado para la obtención del título de Abogado, el cual se encuentra disponible en < https://bit.ly/2LSJTF3>.

** Miembro e investigador de la Fundación Arkhé. Abogado por la Universidad Católica Santiago de Guayaquil. Correo electrónico: fernando.bajana@cu.ucsg.edu.ec/fbajana95@gmail.com

F. BAJAÑA Tovar, "El problema de la corrección en el Arbitraje en Equidad: una propuesta de modelo prescriptivo", Revista Ecuatoriana de Arbitraje, No. 10, 2019, pp. 81-112. 
lo define como la modalidad resolutiva por default. No obstante, a contrariedad de la relevancia normativa que tiene el mecanismo ex aequo et bono, su desarrollo dogmático ha sido muy escaso. En tal virtud, el presente estudio pretende configurar un modelo prescriptivo de laudos equitativos, por medio del cual se defienda una tesis liberal del arbitraje en equidad, valiéndose para eso de criterios y principios económicos como los de eficiencia y de minimización de costos de transacción.

Palabras Clave: arbitraje en equidad, modelo prescriptivo, corrección, razones operativas, eficiencia económica.

\title{
Correctness problem in Arbitration in Equity: an approach to a prescriptive model
}

\begin{abstract}
The Ecuadorian arbitral regime establishes two possibilities for the content of its decisions: (i) foundation in law, based on legislative, jurisprudential and doctrinal premises; and (ii) an argument inspired by equity, where general standards such as fair 'knowing and understanding' and reasonable evaluation are combined. It is this last criterion, of apparent ethical and philosophical inspirations, that engraves within the arbitration a greater normative relevance, insofar as the arbitral law defines it as the default resolution modality. However, contrary to the normative relevance of the mechanism ex aequo et bono, its dogmatic development has been very limited, to this extent the present study is intended to shape a prescriptive model of fair awards, by means of which a liberal thesis of arbitration in equity is defended, by using the principle of economic efficiency and the transaction costs.
\end{abstract}

KeYwords: arbitration in equity, prescriptive model, correction, operational reasons, economic efficiency. 


\section{INTRODUCCIÓN}

La práctica arbitral ecuatoriana admite dos alternativas para la estructuración de sus decisiones: (i) una cuya base se asienta en la aplicación teórico-ideológica del derecho; y, (ii) otra cuyo hilo argumentativo gira entorno a la idea de la equidad. Mientras que en el primer caso los árbitros expiden sus laudos basados "en la ley, los principios universales del derecho, la jurisprudencia y la doctrina"1; para el caso de los laudos en Equidad la ley instituye una remisión prioritaria hacia el leal saber y entender de los componedores.

No obstante, independientemente de la alternativa elegida para la resolución de la controversia, uno de los principales asuntos que subyace en los estudios y reflexiones que se realizan sobre la materia es el relacionado con la idea de la corrección, esto es, el de cuándo se debe entender que una decisión ha sido correcta ${ }^{2}$.

A modo de respuesta, la doctrina ha preferido centrar sus análisis en la comprensión de la relación que existe entre los criterios de corrección y algunos aspectos teóricos del derecho relacionadosconsu conceptualización, y sus procesosdeaplicación, interpretación y argumentación. De estos enfoques, que, si bien han tenido como objeto principal el escrutinio de las decisiones judiciales, se han muchas de sus conclusiones en el tratamiento de los laudos adoptados en Derecho, sacando provecho del alto grado de convergencia estructural que comparten estas dos formas resolutivas, en la medida de que tanto sentencias como laudos ex iure se encuentras inspiradas profundamente en un modelo aristotélico de acople.

1. Ley de Arbitraje y Mediación (LAM), Art. 3, RO No. 145, 4/9/1997.

2. Este tipo de interrogante se puede presentar tanto de una forma abstracta y a priori -¿qué debe comprenderse por una decisión correcta?-, como de manera concreta y a posteriori -¿la decisión adoptada ha sido correcta?-. 
Sin embargo, diferentemente de lo que se suscita entre sentencias y laudos en derecho, en lo que respecta a las decisiones en equidad, resulta poco factible replicar esta adaptabilidad de conclusiones; en primer lugar, producto de la complejidad que se evidencia para reproducir en modelos de resolución equitativa, un esquema clásico de subsunción de supuestos fácticos y enunciados normativos, partiendo desde prescindencia al menos teórica de normas regulativas que sirvan como premisa mayor.

Es así como, frente a la problemática que plantea la dificultad para adaptar los esquemas resolutivos típicos a la esfera específica del arbitraje en equidad, que el autor mediante el presente ensayo ha procurado bosquejar un camino prescriptivo para dar una posible respuesta a la interrogante de la corrección de los laudos arbitrales adoptados en equidad. Para ello, se diseñará y propondrá un modelo de corrección que facilite la comprensión autónoma de los elementos que confluyen en la argumentación de este tipo de decisiones; a los efectos se dedicará un énfasis especial a la explicación del funcionamiento de razones operativas extrasistemáticas de corte económico, y se acogerá una concepción material de la argumentación jurídica.

\section{Presupuestos Conceptuales}

Para iniciar esta exposición, resulta ineludible fijar ciertos presupuestos conceptuales que respalden una construcción narrativa armónica del modelo prescriptivo que se ensayará. De este modo, se comenzará por precisar el objeto de estudio al que se hace mención cuando se utiliza el vocablo modelo; y, la función semántica que ocupa el enunciado corrección respecto de este. Seguido a ello, se examinará la categoría de decisión en su sentido estricto, a fin de descubrir aquellas estructuras que organizan su anatomía y la forma en la que los criterios de corrección la califican. 


\subsection{Un modelo prescriptivo}

Es bien conocido que la palabra modelo no sólo se aprovecha en un marco científico o doctrinal, siendo viable que se la escuche de forma indiscriminada y con una misma incidencia, tanto en conversaciones triviales como en contextos versados, dotándosele en cada caso de un significado distinto. En efecto, como lo retrata Moreno VILLA33, la versatilidad semántica del vocablo modelo llega a tal punto que es plenamente posible que dicha palabra se emplea para apuntar tanto a personas que se tambalean sobre pasarelas, como a esquemas de investigación o de representación de la realidad.

Por lo expuesto, y, con la finalidad de superar la connatural ambigüedad que esta palabra supone, es menester puntualizar que en lo venidero, en aras de prevenir cualquier tipo de confusión, a dicha palabra se le utilizará de conformidad con el significado con el que mayoritariamente se la identifica en el lenguaje de las ciencias; esto es, el de un "arquetipo o paradigma que posibilita una interpretación correcta a un sistema de axiomas, o, más genéricamente, la forma ejemplar que nos proponemos seguir para lograr la acabada ejecución de un proyecto [...]"4.

Pero incluso bajo su definición científica es posible percatar una pretensión de singularización fallida, en fin, la definición que generalmente adoptan los relatos científicos sirve para reconocer a dos objetos diferentes. Como una posible solución a este problema, la epistemología le ha reconocido al concepto de modelo la derivación de al menos dos aplicaciones o funciones distintas. Así, por un lado, se habla de modelos, en el sentido de medio para "interpretación de hechos"; $y$, por otro; se aprovecha la palabra para representar una "formatización ejemplificativa" de alguna conducta determinada.

3. M. Moreno Villa, Filosofia: Filosofia del Lenguaje, Lógica, Filosofia de la Ciencia y Metafisica, Vol. I, Editorial MAD, 2003, p. 298.

4. Íbidem. 
Esta dualidad semántica, ha servido para que seestandarice una subclasificación operativa de la categoría modelo, cuyo parámetro discriminador está dado por la operatividad o función específica que cada modelo desarrolla. A efectos de esto, se tiene modelos: (i) que actúan como esquemas que a través del lenguaje describen hechos de la realidad -idea de modelo como descripción-; y otros, (ii) que se encuentran dirigidos a hacer valoraciones $\mathrm{y} / \mathrm{o}$ construir directrices prescriptivas para un ámbito específico -idea de modelo como axioma-.

Jerzy WrobleWSKI, uno de los primeros en adaptar esta tipología a las necesidades del Derecho, a mediados del siglo XX, agregó a esta subclasificación una tercera, la de los modelos de utilidad mixta, que servirían para poder responder conjuntamente a necesidades de descripción y normalización en un estudio. Posteriormente, ComANDUCCI se inspiraría en el trabajo de WrobLEWSKI y advertiría en este una importante aptitud para el abordaje analítico de los razonamientos jurídicos, toda vez que las perspectivas específicas que aporta configuran una taxonomía tripartita -a las que él llamará aproximaciones- que facultaría a la doctrina a adoptar modelos descriptivos, prescriptivos y teóricos en el estudio de resoluciones y sentencias 5 .

Así, bajo las conclusiones de Comanducci, un modelo obedecerá a una aproximación descriptiva, cuando la pretensión de quien lo desarrolla es meramente narrativa. Aquello implica que quien lo usa se abstendrá a dar el detalle de las fases o actividades que el juez, $\mathrm{u}$ operador jurisdiccional, ha dedicado o dedica para la toma de una decisión específica; sea que se entienda al razonamiento jurídico como un circuito de actividades cognoscitivas, o, como una descripción valorativa de documentos probatorios y legales.

5. F. Carbonell Bellolio, "Elementos para un modelo de decisión judicial correcta", Revista de Estudios de la Justicia, 2017. 
Desde otro enfoque, los modelos teóricos configurarán representaciones simplificadas del razonamiento judicial, construidas a partir de ciertas variables de investigación que el autor elige libremente. BARBERIS señala, al respecto, que estos modelos se exteriorizan cuando el objetivo del investigador es el de conseguir una suerte de abstracción simplificada, alternativa y funcional del razonamiento de un juez ${ }^{6}$.

Finalmente, en lo que atiene a los modelos prescriptivos, aquellos no buscarían la descripción de un acto de decisión jurídica, o su explicación simplificada, sino que tendrían como finalidad el levantamiento de un juicio valorativo sobre la forma de decidir con una consecuente propuesta de directiva, enmarcada en la forma de un prototipo resolutivo9. En ese aspecto, lo que diferencia a las distintas aproximaciones una con otra, es que entretanto "los modelos normativos de pensamiento especifican el ideal [...]. Los modelos descriptivos especifican lo que la gente de una determinada cultura -en este caso jurídica- hace realmente y cómo se desvían de los modelos normativos $[\ldots]^{\prime \prime 10}$.

Así las cosas, si regresamos la atención al objeto que se pretende modelar, esto es, las decisiones arbitrales en equidad, se vislumbra que existen al menos dos razones de orden

6. BARBERIS insiste en que por medio de los modelos teóricos se alcanza una representación (i) simplificada de la realidad, en la medida de que tan solo se atenderán los parámetros importantes para el hecho jurídico en estudio. Por otra parte, declara que (ii) el carácter funcional, vendría dado por el hecho de que se construyen para cumplir con fines específicos, sean cognitivos o directivos, que reportan utilidad para el autor del modelo. Finalmente, la alternatividad, de los modelos teóricos, la evalúa en su capacidad de reconstruirse según los parámetros que se escojan en cada ocasión.

7. M. BARBERIS, Filosofia del diritto: Un'introduzione teorica, Giappichelli, 2000.

8. No todos los autores concuerdan en identificar a los modelos normativos con los modelos prescriptivos, destacando de entre aquellos Gabuccio Cerezo, para quien si bien es cierto que mediante ambos modelos se busca dar respuesta a la pregunta ¿cómo deberíamos pensar? Los modelos prescriptivos se limitarían a "prescribir, en el sentido de recomendar, algunos modos de razonamiento o de enfoque particularmente apropiados a una determinada actividad" sin que se "trate de que haya únicamente una manera de hacer o de plantearlo". Mientras que por su parte los modelos normativos, pretendieron ser la única solución a dicha interrogante "son modelos que definen el mejor modo de pensar para lograr unos objetivos determinados".

9. P. Comanducci, Razonamiento jurídico: Elementos para un modelo, Fontamara, 1999.

10. J. Baron, Thinking and deciding, Cambridge University Press, 1983. 
lógico y práctico para preferir que en su modelaje se acate una aproximación prescriptiva antes que a una teórica o descriptiva.

De esta manera, una primera razón para la preferencia de esta alternativa viene dada por el hecho de que (i) levanta poco interés investigativo y muy baja utilidad doctrinal centrarse a recrear un modelo de descripción de decisiones arbitrales en equidad. Hacerlo implicaría limitarse, en exclusiva, a relatar de forma puntual las actividades que han desarrollado los árbitros en el proceso del laudaje equitativo. Empero, a diferencia de la prevalencia que tienen los modelos descriptivos en las decisiones judiciales, cuya utilidad se puede ver en los repositorios de jurisprudencia, gacetas judiciales, entre otros; en el caso del arbitraje, aquellos no traducen mayor provecho para el desarrollo jurídico. Para esto se debe entender que tales narraciones, sobre la manera en la que los árbitros resolvieron determinado tipo de casos, dentro de un contexto histórico y geográfico específico, no podrán ser luego utilizadas como precedentes vinculantes o normas sistémicas para la resolución de otros casos, debido a la falta de unidad jurisdiccional, que caracteriza a los sistemas arbitrales. Esta eventualidad resulta plenamente impráctica, más aún si se considera la escasez, sobre todo en el contexto doméstico, de procedimientos arbitrales resueltos propiamente en equidad ${ }^{11}$. A lo que habrá que agregar el carácter reservado con el que se hallan protegidos muchos de estos procedimientos, lo cual dificultaría de sobremanera la posibilidad de descripción de dichos casos sin la afectación de derechos de terceros ${ }^{12}$.

11. Así lo afirma Ana María LARREA, quien ha señalado: "estadísticamente, la mayoría de los arbitrajes administrados que se han sustanciado en el país, tomando como base las cifras de los Centros de Conciliación y Arbitraje de Guayaquil y Quito, han sido en derecho y no en equidad [...]. En consecuencia, los abogados no hemos tenido mayor contacto práctico con la aplicación de la equidad judicial o arbitral, lo que me lleva a concluir que, el que abiertamente se prefiera un arbitraje en derecho es el desconocimiento de lo que es la equidad, o la percepción de ser esta ajena u opuesta al derecho, y de consecuencias imprevisibles".

A. M. LarReA, "Alcance y límites del arbitraje en equidad", Revista Jurídica Online, 2011, pp. 22-23.

12. En efecto, el artículo 34 de la LAM establece que: "Las partes sin perjuicio de los derechos de terceros, podrán convenir en la confidencialidad del procedimiento arbitral [...]". Norma semejante se lee, en el contexto del arbitraje internacional, en los artículos 28(3) y 34(5) del 
En esta misma línea, (ii) es de poco provecho el desarrollo de un modelo teórico de decisiones en equidad, si se percata la carencia de elementos sustanciales para su construcción, esto como resultado de la conceptualización precaria y los esquemas residuales de interpretación y aplicación que imperan en la doctrina. Por tales consideraciones, se ha de preferir focalizar el presente estudio en la edificación de un modelo prescriptivo para la argumentación de laudos en equidad, que sirvan de directiva metodológica y referente axiológico a los operadores heterocompositivos.

De ahí que el contenido del presente ensayo será principalmente ideológico, entendiendo por ideología lo que la Filosofía Analítica instruye de esta, o sea, una referencia al deber ser antes que al ser de las cosas. Pues como bien se conoce, esta escuela filosófica diferencia la ideología de la teoría, por el hecho de que, mientras "la teoría versa sobre aquello que es y que puede ser objeto de descripción por parte de un individuo; la ideología versa sobre aquello que debe ser y cumple una función normativa que aspira a dirigir el comportamiento de los árbitros- hacia la realización de determinados valores -la equidad-"13. Siguiendo esta concepción es factible afirmar que el carácter prescriptivo del modelo que se propondrá, en última instancia, dibuja un paradigma o guía del deber ser para los laudos en equidad; debiéndose derivadamente interpretar, como prescripciones axiológicas, cada una de las consideraciones que se expondrán adelante.

\subsection{La idea de corrección y su adopción en el presente modelo}

De manera semejante a lo que se suscita con el vocablo modelo, la función semántica de la palabra corrección se suele

Reglamento de Arbitraje de la Comisión de las Naciones Unidas para el Derecho Mercantil Internacional.

13. F. Carbonell Bellolio, N. 5, p. 11. 
tomar como consabida y no problemática. Que si tal acción fue correcta, o, que si tal no lo fue, constituyen afirmaciones que se oyen de modo cotidiano, sin que necesariamente en alguno de los casos, el hablante se moleste en confirmar la función que intenta desprender del ámbito de sus aseveraciones.

En este sentido, para entender la idea de corrección, algunos autores recomiendan concentrarse en el contexto en el que la palabra se emite y la relación que ocupa con las cosas circundantes u objetos de recepción. De esta manera, no es lo mismo hablar de corrección en un contexto concreto, que hablar de corrección de forma abstracta o genérica.

Bajo este ánimo ilativo, en su acepción genérica, se emplearía la locución corrección, en la forma del adjetivo correcto o correcta, para calificar positivamente o posicionar un signo de aprobación sobre una determinada conducta, hecho o descripción ${ }^{14}$.

Así las cosas, si bien es irrelevante para el uso genérico de este vocablo, el objeto sobre el cual se está desplegando el juicio de corrección (da lo mismo si se hace sobre un juicio de valor, comentario o descripción), se puede prever que tiene más sentido que aquel se desarrolle sobre ciertas cosas por sobre otras; por citar un ejemplo, tiene mucha más lógica la expresión "la política de sanidad de la República Federal Alemana es correcta", y no así la afirmación "la montaña y el río son correctos". Esto se debe a que, por lo general, se acostumbra a dirigir el atributo corrección -o de verdadero, o certero- para cualificar hechos en los que ha intervenido la actividad racional del hombre ${ }^{15}$.

14. Así lo ha expresado CARBonell Bellolio, al manifestar: "Por otra parte, el atributo "correcta/o" se aplica a diversas cosas, tales como enunciados o afirmaciones, acciones, conductas, normas y decisiones. Respecto de todas estas cosas existe intervención humana en alguna medida; por el contrario, estos términos no se emplean para calificar directamente eventos de la naturaleza, careciendo de sentido expresiones tales como "aquel terremoto es incorrecto" o "es correcto que hoy llueva". Ibídem.

15. Debido a que "las acciones, normas o decisiones tienen en común que son productos humanos susceptibles de evaluación en tanto conllevan una toma de posición ético-normativa". Ídem, p. 6. 
Luego, desde una perspectiva abstracta del criterio de corrección, es viable notar que, desde este ángulo, la palabra sometida a estudio carece por sí sola de un contenido propio, es decir, que no refiere per se a categoría ideal o concepto alguno, limitándose a actuar como un contenido estructural. En palabras más sencillas, abstrayendo su aplicación de los casos concretos, la palabra corrección no tiene significado alguno, por cuanto solo es posible desprendérsele cierta connotación, cuando va acompañada de un objeto al que calificar, y de un parámetro prefijado que sirva como criterio de contraste o correspondencia. Así, resulta más acertado abordar a la corrección, en su concepción abstracta, como un señalizador de la existencia o ausencia de una relación de ajuste entre enunciados específicos y variables de contraste. De ahí que sólo se reporte un resultado positivo, y por lo tanto se diga que un objeto es correcto, cuando se reproduzca una relación de correspondencia entre dicho objeto y un sustrato, que generalmente corresponde a una tesis de la verdad ${ }^{16}{ }^{17}$.

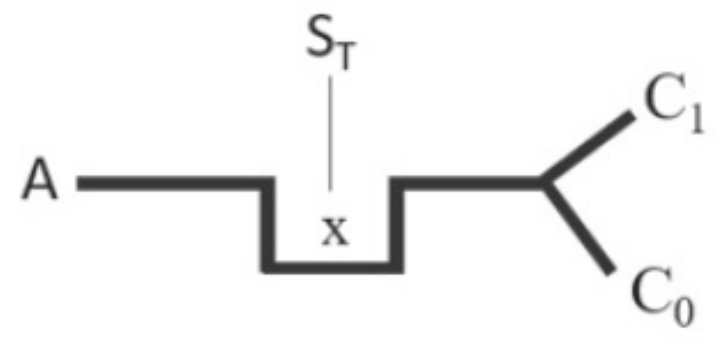

Figura 1: Representación abstracta de un juicio de corrección ${ }^{18}$

16. Bajo esta línea argumental, diremos que la palabra corrección, "funciona como un designador flexible de una relación de ajuste de cualquier contenido epistémico o axiológico -esto es, de una relación que se da entre diversas cosas y diversos parámetros- que se valora positivamente por quien emite el juicio". Ídem, p. 3.

17. Por consiguiente, si se actúa desde una teoría objetiva de la realidad, un enunciado descriptivo será correcto si lo dicho se corresponde con lo que se encuentra presente en el mundo exterior; pero si se emplea como parámetro de contraste a una teoría coherensionista, la corrección de un enunciado dependerá de la congruencia que guarde con el resto de los enunciados que conforman un sistema. Por lo que se puede ver que el resultado del juicio de corrección varía en cada caso, según el sustrato teórico que se le aporte.

18. Se observa que el juicio corrección desde una perspectiva abstracta, funciona como un contenido estructural, donde la corrección $(\mathrm{C} 1)$ o incorrección $(\mathrm{C} 0)$ de un objeto $(\mathrm{A})$ depende de su 
Mas ninguno de estos enfoques son lo suficientemente útiles para el ámbito del estudio propuesto, por cuanto al no perseguirse la creación de un modelo de decisión que se limita a calificar de forma vaga la apariencia de positividad o negatividad de un razonamiento jurisdiccional -pretensión aprobatoria-, es absurdo concentrarnos en un enfoque genérico del juicio de corrección; y, como se vio al final, un enfoque abstracto resulta inerte mientras no se le otorgue un sustrato teórico que sirva como elemento de contraste para dicho juicio.

Frente a esto, un planteamiento concreto del juicio de corrección, evidencia ser la salida más acertada para la calificación de las decisiones arbitrales en equidad. No obstante, una concepción concreta exige la determinación previa del objeto a calificarse, en este caso, la decisión en sentido estricto, y el sustrato teórico que fungirá de parámetro o estándar de calificación. Como consecuencia de lo expuesto, se pasará a definir lo entendido por decisión arbitral -objeto de calificación-, con un especial repaso en los laudos en equidad; mientras que en lo relativo al sustrato de contraste, aquel se expondrá gradualmente como una identificación al criterio económico de eficiencia.

\subsection{El objeto del juicio de corrección: la decisión en equidad}

Bajo la categoría de decisión jurisdiccional es posible denotar tanto: (i) la serie de pasos que constituyen el procedimiento que un operador jurídico despliega a la hora de resolver una controversia; como, (ii) el resultado final que se desprende de dicho procedimiento. Por consiguiente, se puede identificar por medio de esta noción:

[...] tanto a la formulación de las partes que componen este procedimiento [premisas justificadas] como el resultado o

correspondencia con un sustrato teórico prefijado (St), que por lo general lo conforma una teoría de la verdad. 
decisión final [conclusión], que se obtiene de la subsunción de la premisa fáctica -cadena de enunciados referidos especialmente a hechos- en la premisa normativa -cadena de enunciados cuyo contenido son especialmente normas $-{ }^{19}$.

Estas dos alternativas de significación se van analizar durante el presente estudio, de manera tal que se pueda averiguar las formas de interacción que se suscitan entre el criterio, o los criterios de "corrección", y cada uno de los componentes/etapas que se hallan al interior de los laudos en equidad, para lo cual se profundizará previamente en la examinación de cada uno de dichos elementos.

Llegado este punto, es necesario destacar que, pese a que ha sido una afirmación constante dentro de la doctrina procesal, la estimación de las decisiones jurisdiccionales como estructuras subsuntivas, que relacionan un elemento de tipo fenomenológico con otro de carga normativa ${ }^{20}$; dicha configuración típica y bipartita, exhibe una serie de complicaciones a la hora de explicar los laudos en equidad, en especial en lo concerniente a dos situaciones. La primera y más evidente, está dada por (i) la obligada prescindencia de normas jurídicas positivas en este tipo de decisiones; lo que a prima facie impide la obtención de una premisa mayor para el silogismo decisorio; y, la segunda, no tan evidente como la primera, consiste en (ii) la imposibilidad de la composición de premisas fácticas -siguiendo el modelo tradicional de selección de hechos-, como una derivación del primer problema, en cuanto la ausencia de normas positivas que procedan como

19. F. Carbonell Bellolio, N. 5, p. 3.

20. Esta es la idea que Atienza ha expuesto, cuando ha indicado que: "Suele decirse que el silogismo subsuntivo, la subsunción, es el esquema general de argumentación en la justificación judicial. $\mathrm{O}$, dicho en otra terminología, constituye la justificación interna del razonamiento judicial. Esto es aproximadamente cierto, en cuanto la premisa normativa [final] de razonamiento judicial es, en general, una regla de acción que tiene la forma: 'si se da las circunstancias o condiciones de aplicación X (un caso genérico), entonces alguien puede, debe o tiene prohibido realizar una determinada acción Y'. A partir de aquí, todo lo que se necesita es clasificar o subsumir cierta realidad fáctica en el supuesto de hecho de la norma.". M. AtIEnZA, Curso de Argumentación Jurídica, Trotta, 2013. p. 183. 
premisas mayores, imposibilita la existencia de un punto de referencialidad para la fácil discriminación y selección de los enunciados fácticos, ${ }^{21}$ los cuales generalmente se escogen a partir de las exigencias probatorias contenidas en la propia norma positiva.

Es este el motivo por el cual, en lo tocante a la forma de dirigir el juicio de corrección sobre los elementos del laudo en equidad, no se seguirá la clásica división formal de razones, en premisas fácticas y premisas normativas, ya que, tal como se va a destacar luego, el presente análisis optará por una concepción material y práctica de la argumentación jurídica, desde la cual es viable operar sin referencia a normas sistémicas y autoritativas, ensayándose para el presente caso, el uso de varios principios de orden axiológico, principalmente de aquellos que los filósofos y teóricos han encasillado dentro de las teorías de la justicia, sobre todo en lo relacionado con los principios de eficiencia y distribución que instruyen Pareto y Coase.

\section{DE LA CONCEPCIÓN FORMAL A LA CONCEPCIÓN MATERIAL DEL RAZONAMIENTO JURÍDICO}

De forma general, los operadores jurisdiccionales, incluyendo jueces y árbitros, fundamentan sus decisiones en enunciados normativos obtenidos a partir de reglas válidas que configuran el sistema jurídico, a los cuales acoplan otros enunciados de carácter descriptivo donde se contienen afirmaciones o negaciones sobre la realidad. Este esquema de pensamiento, que se conoce como silogístico o subsuntivo, constituye el exponente principal de la concepción lógica de la

21. A pesar de que la obtención de premisas fácticas puede parecer un grave problema desde una perspectiva clásica de toma de decisiones, producto de la ausencia de normas positivas que puedan utilizarse como criterio de discriminación y selección de hechos; dicha idea pierde peso si se considera que el lugar de las premisas normativas dadas por reglas de acción puede ser suplido por nomas extrasistémicas, e inclusive por algunas normas con rasgos sistémicos particulares, como lo sería por ejemplo la fuente de la controversia, $v$.gr. el contrato infringido. 
argumentación jurídica, concepción cuya centralidad gira en torno a las inferencias formales.

Ora, como instruyen Atienza y Ruiz MANero este modelo argumentativo no puede siempre ser recreado en las decisiones, ya que al hallarse supeditado su funcionamiento a la existencia de reglas de acción, esto es, "pautas específicas de conducta que establecen mandatos o permisiones que se caracterizan porque [...] -y que- su estructura consiste en un antecedente o condición de aplicación, que contienen un conjunto cerrado de propiedades; y un consecuente o solución normativa en donde cabe distinguir, a su vez, dos elementos: una acción y su calificación deóntica" ${ }^{22}$, cuando dicha clase de normas se hallen ausentes o impedidas de utilizarse, como en los casos de derrotabilidad de reglas o expresa prohibición, la aplicación de dicho arquetipo resultará frustrada ${ }^{23}$.

En este sentido, el levantamiento de un análisis sobre el segundo y tercer inciso del artículo 3 de la Ley de Arbitraje y Mediación (en adelante, LAM), nos permitirá admitir o rechazar la presencia de una situación de derrotabilidad de normas sistémicas -en especial de reglas de acción-, y detectar así la oportunidad o improcedencia de la aplicación del modelo lógico subsuntivo al interior del arbitraje en equidad. La norma precitada, sanciona: "Si el laudo debe expedirse fundado en la equidad, los árbitros actuarán conforme a su leal saber y entender y atendiendo a los principios de la sana crítica. [...] Si el laudo debe expedirse fundado en derecho, los árbitros deberán atenerse a la ley [...]"24. Texto

22. M. Atienza \& J. Ruiz Manero, "Rules and Principles Revisited", Associations Journal for Legal and Social Theory, Vol. 4, No. 1, 2000. p. 9.

23. En efecto, puede presentar el caso de que las reglas que versen sobre la materia de la controversia estén expuestas siguiendo un patrón finalista y no de acción, donde el consecuente no expresa una orden, prohibición o permisión conductual específica, sino que su contenido deóntico contiene la exigencia de alcanzar cierto estado de la realidad -cumplir con cierto fin-. Asimismo, se puede presenciar situaciones donde los operadores jurisdiccionales tan solo cuenten con principios.

24. LAM, Art. 3(2)(3), RO No. 447. 20/12/2006. 
del cual se pueden extraer al menos dos interpretaciones disímiles.

La interpretación más extendida, y con mayor acogida en la práctica arbitral, sostiene que la norma transcrita no precisa una diferenciación insuperable o disyunción excluyente entre dos sistemas arbitrales, sino que más bien expone una relación copulativa entre el arbitraje ex aequo et bono y el stricti iruis -interpretación conservadora-. Por consiguiente, para quienes defienden esta idea, en el arbitraje en equidad, el hetercomponedor no está compelido a decidir en desatención de las fuentes clásicas del derecho, más bien tendría la obligación de hacerlo. Por ende, el carácter equitativo del laudo desde esta perspectiva, gravita exclusivamente en la contingencia de que los árbitros realicen una interpretación más flexible de la norma, ante su vacío o ambigüedad.

Entre los tratadistas ecuatorianos ha sido esta tendencia, de corte conservador, la que más ha prevalecido, siendo un ejemplo paradigmático de esta tendencia, la defensa que Ana María LARREA ha realizado sobre la misma, cuando ha afirmado que:

[...] debemos colegir, que el fallo en equidad parte siempre del derecho, y solo de encontrarse que las disposiciones aplicables al caso resultan injustas, o existe vacío legal, procedería -el árbitro- a buscar la solución justa que el legislador se habría planteado, teniendo presente el objetivo de la ley, para ese mismo caso concreto, y según su conciencia ${ }^{25}$.

Por otra parte, la segunda varianteinterpretativa del artículo precitado -tesis liberal-propone un sentido totalmente opuesto de entenderlo, por cuanto, para dicha modalidad interpretativa, el legislador al momento de hacer la categorización por la que distingue entre dos paradigmas de resolución arbitral: en equidad y en derecho, ha buscado edificar dos esquemas excluyentes de decisión cuyas modalidades argumentativas

25. A. M. Larrea, N. 11, p. 36. 
son incompatibles. Así pues, mientras en el arbitraje en derecho lo primordial para la justificación del laudo es la alusión a normas sistémicas; en el arbitraje en equidad, lo conveniente sería recurrir a razones o premisas de fuera del sistema jurídico, o, dicho de otra forma, el estructurar un razonamiento a partir de premisas no autoritativas.

Por consiguiente, la falta de normas sistémicas en el arbitraje en equidad -máxima que defiende la segunda tendencia- colocaría en jaque a la funcionalidad de los modelos de razonamiento con bases silogísticas, toda vez que, al llevarse acabo de toda la operación de decisión en ausencia de normas regulativas, no habría elementos a los que se les puede atribuir la calidad de premisas mayores, pieza clave en los procesos de silogismo arbitral. Se advierte, entonces, por contraste, que un modelo argumentativo formal solo podría funcionar al interior del arbitraje en equidad, bajo una interpretación conservadora del artículo 3 de la $\mathrm{LAM}^{26}$, en la medida que únicamente bajo este esquema se les impele a los árbitros la referencialidad a razones sistémicas.

Ante lo expuesto, es un deber del autor discernir entre las dos corrientes expuestas, con objeto de esclarecer si la concepción argumentativa en la cual amparará su modelo prescriptivo apelará a una representación lógico inferencial; o más bien, a alguna especie de lo que la doctrina denomina lógica material o argumentación práctica, donde la centralidad de la forma y las inferencias es suplida por la medición de la fuerza y relevancia de las razones que se ofertan. En su tarea, el autor abonará cuatro razones para preferir una noción liberal del arbitraje equitativo sobre una conservadora, rechazando los argumentos brindados por la doctrina mayoritaria para la defensa de esta última alternativa.

26. LAM, N. 24, Art. 3. 
Entonces así se puede ver, en primer lugar que, (i) carece de propósito que el legislador, al momento de establecer una norma que instaure dos alternativas para la elaboración de laudos, simplemente haya deseado que dicha categorización se tome por inútil, en el sentido de que las estructuras y razones de un modelo arbitral, ya sea en derecho o en equidad, al final resulten libremente endosables y utilizables en otro. En fin, si en el arbitraje en equidad se debería partir aplicando las mismas estructuras que se utilizan en el arbitraje en derecho, tal como lo afirma la interpretación conservadora del artículo 3 de la LAM, se estaría admitiendo una aplicación conjunta del modelo equitativo y el jurídico stricti iuris, lo que a su vez implica, que la relación entre dichos modelos sería de tipo copulativa, es decir incluyente. No obstante, si esta hubiera sido la intención del legislador, ¿acaso no hubiera sido más útil que aquel construya una sola regla que mande que en los litigios arbitrales se resuelva aplicando tanto el derecho como la equidad?

Colegida de la primera razón, (ii) la mentada relación copulativa que habría entre arbitraje en derecho y arbitraje en equidad, tal como lo instruye la escuela conservadora, adolece en el fondo de una unidireccionalidad injustificada. Lo dicho se observa por cuanto, si bien los defensores de la corriente conservadora abogan para que en el arbitraje en equidad se apliquen normas del derecho positivo, no siempre consienten una funcionalidad inversa de esta maniobra; es decir que, en el caso de arbitrajes en derecho, se motiven los laudos en "principios de equidad", como lógicamente tendría que suceder si se estuviera frente a una relación lógica de copulación o inclusión.

Desde esta perspectiva, (iii) tampoco resulta del todo convincente que el arbitraje en equidad sea entendido como una variante en la cual el árbitro está permitido de corregir la sobreinclusión o infrainclusión de las reglas (interpretación flexible), por cuanto dicha conclusión dotaría a la modalidad 
ex aequo et bono, de un carácter residual y supletorio, al comprender que sólo habría de operar cuando las reglas jurídicas posean generalizaciones inadecuadas, o en otras palabras, cuando una "regla no incluye casos que deberían incluirse o porque incluye casos que no deberían incluirse" ${ }^{27}$. Esta idea podría llevar a pensar que únicamente en los casos donde las partes hubiesen pactado un arbitraje en equidad, es factible corregir la distorsión deóntica de las normas regulativas, hallándose, por ende, dicha posibilidad vetada de los arbitrajes en derecho. Pero, ¿acaso no es posible que en un arbitraje en derecho las reglas a aplicarse adolezcan de sobreinclusión o infrainclusión?

Para finalizar, (iv) la promoción de la tendencia conservadora terminaría contrariando a los principios más íntimos de la institución arbitral; en efecto, el principio de la autonomía de la voluntad de las partes terminaría comprometido. Si el convenio de las partes sobre una alternativa resolutiva en específico no interfiere en nada para que los árbitros apliquen estructuras de las dos e inclusive de la otra, ¿cuál es el sentido de este principio?

Por todo lo expuesto, el autor concluye que lo más apropiado en pro de una mayor autonomía teórica y metodológica para el arbitraje en equidad lo es la defensa de un enfoque liberal de aplicación, mediante el cual se vislumbre a esta modalidad resolutiva como un esquema independiente de decisión, que no se halla subordinado al tipo de premisas formales y sistémicas que se emplean clásicamente en los laudos adoptados en derecho y las decisiones judiciales.

En consecuencia, al momento de decidir, en el arbitraje en equidad, el razonamiento decisorio no deberá recrear una secuencia de proposiciones o enunciados lingüísticos

27. M. ATIENZA, N. 20, p. 298. 
dispuestos según una forma específica (silogismo categórico, modus ponens, etc.); en su lugar, la actuación argumentativa de los árbitros deberá acoger una concepción material y práctica, en virtud de la cual, el elemento esencial de la decisión judicial lo constituirán razones sustantivas, moderadas según su fuerza y relevancia, antes que por su forma. Como producto de esto, "el centro [de la argumentación] se situará entonces, en las premisas, pero no entendidas en cuanto enunciados con una cierta forma, pues lo que interesa aquí es precisamente, su contenido de verdad y de corrección: la premisa" ${ }^{28}$.

\subsection{Razones sustantivas}

Para empezar, tal como se sostuvo antes, el principal signo distintivo del arbitraje en equidad está dado por un supuesto de derrotabilidad radical de reglas, el cual se origina siempre que "de acuerdo con el derecho, concurren razones para basar la respuesta a un problema de indeterminación en criterios extrajurídicos, es decir, criterios ajenos a los que el propio derecho establece" ${ }^{29}$. Esta última idea, inferida de la interpretación liberal del segundo y el tercer inciso del artículo 3 de la LAM.

En virtud de esta noción, para que un determinado argumento o premisa pueda ser tomado en cuenta dentro de un ejercicio resolutivo en equidad, esta debe estar dotada de dos propiedades esenciales; a saber: (i) poseer la aptitud para ser utilizadas en contexto argumentativo material y práctico; y, además, (ii) poder contestar al tipo específico de razones que se conoce como extrasistémicas.

Ahora bien, aunque a prima facie pueda aparentarlo, el uso de premisas extrasistémicas en decisiones jurisdiccionales,

28. Ibíd, p. 275.

29. A. Rodenas Calatayud, "En la penumbra: indeterminación, derrotabilidad y aplicación judicial de normas", DOXA, No. 24, 2001, p. 77. 
inclusive en sede judicial, está lejos de ser un fenómeno novedoso para el mundo del derecho, y, a contrario sensu, como bien señala ATIENZA: "en los argumentos jurídicos, en sentido amplio, algunas de las premisas que figuran en los mismos provienen del propio sistema jurídico, son enunciados del sistema jurídico, mientras que otras son extrasistemáticas [... $]^{\prime 30}$. Sin embargo, lo particular del modelo que se propone es que, en aquel, a diferencia de lo sucedido al interior de las decisiones judiciales con los enunciados extrasistémicos, donde ocupan el lugar de premisas fácticas, para el presente caso, cumplirán un funcionamiento semejante al de una premisa normativa. Sobre esto, y antes de avanzar al detalle de las razones que se usarán en nuestro modelo prescriptivo, es conveniente anotar que el mantenimiento de la clásica división de las premisas, entre fácticas y normativas, poco o nada aportan en la presente situación. Y es que, como se dijo antes, el clásico acople silogístico de premisas menores -fácticas- en premisa mayores -normativas-, no se reproduce dentro de un contexto material y práctico de argumentación jurídica, como el que se ha defendido. Así las cosas, resulta más prudente valerse de una clasificación como la de RAz para quien las razones, sin importar su carácter descriptivo o deóntico, deben clasificarse según la fuerza o relevancia que ocupan al interior de un razonamiento, en operativas y auxiliares; de tal modo, las primeras configuran "la premisa del deber ser, que en sí misma podría constituir una razón completa para alguna acción" 31 , por su parte configuran "un juicio fáctico que indica un medio para satisfacer una razón operativa" ${ }^{32}$.

Dada esta diferenciación, y en consonancia con el objeto de este estudio, el autor se limitará a prescribir razones de tipo operativo, considerando que, si bien es factible establecer un mandato deóntico general aplicable a todos los casos de

30. M. AtienZa. N. 20, p. 284.

31. J. RAz, Razón práctica y normas, CEC, 1991, p. 189.

32. Ibídem. 
decisiones en equidad, lo mismo no puede suceder con las premisas auxiliares, ya que las razones auxiliares o de soporte que se otorgan para la justificación de un laudo, cambiarán según los hechos particulares que motivan cada controversia, imposibilitando su prescripción generalizada.

\subsection{El juicio de corrección: las razones y la decisión en equidad}

Frente a este escenario, y recordando que, de conformidad a lo tratado previamente, los laudos en equidad deben abordarse bajo una concepción material de la argumentación, según la cual "las cuestiones prácticas se responden con razones no autoritativas, las cuales comprenden tanto razones morales, como éticas y pragmáticas" ${ }^{\prime 3}$. Se había explicado el por qué de la necesidad de basar las decisiones de los laudos en equidad en premisas sustantivas, esto es, razones que "no supongan apelaciones a la autoridad [y que] [...] consistan en consideraciones morales, económicas, políticas o de otro carácter social" ${ }^{34}$. Por tales motivos, en el presente ensayo se probará cómo el criterio paretiano de eficiencia puede constituir un importante pilar argumentativo para este tipo de decisiones.

Desde esta óptica, el óptimo de Pareto cumpliría dos funciones específicas dentro de nuestro modelo prescriptivo. Así, cumple las veces de: (i) una razón operativa, cuyo contenido normativo, descrito con la fórmula de ajuste mundo a lenguaje, establece que: "los árbitros en su decisión deben alcanzar una distribución eficiente entre los intereses económicos de ambas partes"; y, (ii) de un sustrato teórico de contraste para la determinación de la corrección de la decisión propiamente dicha, y el resto de razones auxiliares.

33. R. Alexy, La construcción de los derechos fundamentales, Ad-Hoc, 2010, p. 38.

34. R. Summers, "Two types of subsuntive reasons: The Core of a Theory of Common Law Justification", Cornell Law Review, No. 63, p. 716. 
En lo que refiere a la primera función, (i) Pareto establece que una asignación de bienes es eficiente cuando no sea posible realizar ninguna modificación de esta que implique una mejora en la situación de algún sujeto, sin el empeoramiento en la de otro $^{35}$; es decir, una asignación $(A)$ es eficiente $(E f)$ si no existe otra posible, que mejore las situaciones de ambas partes, $\mathrm{o}$, por lo menos, la de una sin dañar la de otra.

Inspirado en esta noción de eficiencia, en nuestro modelo prescriptivo de laudo en Equidad, el criterio de asignación $(A)$ es sustituido por el de decisión arbitral $(D A)$, y las variables de bienes y eficiencia son suplidas por las de interés procesales (IP) y corrección de laudo (CL). Consecuentemente, lo que se busca mediante esta relectura del óptimo de Pareto y su adaptación al mundo arbitral, es la comprensión de las decisiones equitativas, como un caso de distribución eficiente de cargas económicas deducidas en los actos procesales.

Para comprender de mejor manera esta noción, lo primordial es partir desde las teorías que explican la naturaleza del proceso, específicamente el grupo que describe a dicha institución como una relación jurídica transmutada, o sea, una relación obligacional privada que ha derivado en una relación procesal de carácter público. De esta forma, las prestaciones recíprocas de la relación original-generalmente contractual-se convierten en los actos procesales de acción y de excepción, más específicamente en sus contenidos económicos. Es esta la razón, para que, de forma general en los procesos jurisdiccionales, $\mathrm{y}$, específicamente en aquellos que se desarrollan dentro del sistema arbitral, donde las materias están vinculadas con pretensiones transigibles, se evidencie que tanto la pretensión que contiene la acción, como la excepción que se le opone en la contestación, engloban intereses para la satisfacción de necesidades materiales, el lucro en el caso del actor y el ahorro

35. K. Case \& R. Fair, Principios de Microeconomía, Pearson, 1997, p. 306. 
en el demandado ${ }^{36}$. De ahí, que el papel del árbitro en equidad, desde esta nueva perspectiva, no sería otro que el de actuar como un tercero imparcial que se encarga de efectuar una distribución eficiente y equilibrada entre los intereses económicos de ambas partes, siendo esta la máxima que pasaría a inspirar toda reflexión efectuada al interior del arbitraje en equidad ${ }^{37}$.

Por citar un ejemplo, en una controversia donde $i$ reclama la solución forzosa de un contrato de compraventa a $j$; la pretensión de $i$ por lo general residirá en hacerse con el pago del bien vendido; por su parte, la excepción natural de $j$ naturalmente será el ahorrarse dicho pago. Lo que evidencia que, en ambos casos, la intención de las partes es la de salvaguardar un interés de utilidad para sí.

No obstante, los intereses económico-procesales de las partes se hallan ligados de forma irrompible, siendo errado observarlos individualmente, con tal suerte que su expresión adopta la forma de una relación inversa, donde a mayor satisfacción del actor hay una menor satisfacción del demandado; frente a este aspecto, el árbitro estaría obligado a alcanzar el más alto nivel de satisfacción de una de las partes sin que implique un daño para la otra.

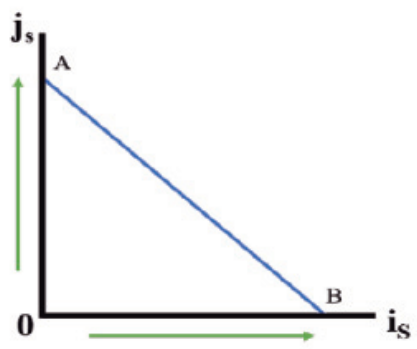

Figura2

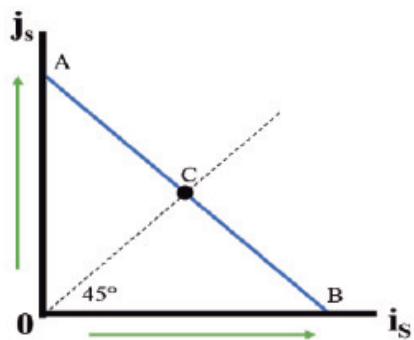

Figura 3

36. El estudio de la satisfacción de necesidades materiales es el objetivo central de la Economía, de ahí la validez metodológica del empleo de criterios propios de dicha ciencia como razones operativas en la resolución de procesos arbitrales. Véase, D. Pedregal, Manual de Microeconomía: Todo lo imprescindible para entenderla, Ediciones Lulú, 2011, p. 2.

37. J. Silva Vallejo, "Los fundamentos científicos del Derecho Procesal”, Themis, No. 14, 1989, p. 25. 
Así, las cosas, como se desprende de la Figura 2, la recta $A B$ tiene una pendiente negativa que demuestra que a mayor satisfacción $(\uparrow)$ del primer sujeto (js) menor es el beneficio $(\leftarrow)$ del segundo sujeto $\left(i_{s}\right)$; pasando lo mismo a la inversa, a mayor satisfacción $(\rightarrow)$ del segundo sujeto $\left(i_{s}\right)$ menor es el beneficio $(\downarrow)$ del primero $\left(j_{s}\right)^{38}$. Por consiguiente, la salida eficiente de distribución en estos casos siempre corresponde a un punto de equilibrio $\mathrm{C}^{39}$, donde la recta $\mathrm{AB}$ es cortada de forma imaginaria por un vector originado a los $45^{\circ}$ del vértice 0 , punto en el cual los intereses de ambos sujetos se encuentran estimados de forma equitativa. RAWLs ha señalado al respecto, que una decisión en la cual "la línea de $45^{\circ}$ indica el lugar de la distribución equitativa [...] supone una interpretación cardinal interpersonal de los ejes"40; de lo que se infiere que un laudo arbitral adoptado en equidad que acoja como referencia cardinal un punto $\mathrm{C}$ de distribución, como razón operativa, además de alcanzar una repartición eficiente de cargas económicas dentro de una relación procesal, responde de forma objetiva a una idea de Equidad, aunque de forma impersonal y objetiva.

En virtud de lo antedicho, la propuesta de la óptima de Pareto como razón operativa de los laudos en equidad, puede vislumbrarse como acertada por su carga teórica y su utilidad práctica. En fin, al funcionar este tipo de resoluciones con exclusión de normas sistémicas, especialmente de reglas de acción, lo que más natural resulta es emplear una norma sustantiva, que observe a la controversia arbitral, no como un ente institucional del derecho, sino como un fenómeno transaccional de la economía, cuya distribución y eliminación de externalidades ha sido puesta a la orden de un tercero imparcial.

38. El eje horizontal representa los intereses de i, por lo tanto, el incremento en satisfacción se mide hacia la derecha alejándose del punto de origen $0^{\circ}$; con j sucede lo propio en el eje vertical, pero de forma ascendente.

39. En adelante se llamará punto $\mathrm{C}$, a un punto ideal de distribución dado a los $45^{\circ}$ de un vector $\mathrm{AB}$.

40. J. Rawls, Teoría de la Justicia, Fondo de Cultura Económica, 2010, p. 75. 
Por otro lado, (ii) en su rol de sustrato teórico de contraste para la determinación de la corrección de las decisiones propiamente dichas y de sus razones auxiliares; el criterio paretiano de eficiencia, funciona de forma semejante a como lo hace el criterio de verdad absoluta o verdad objetiva de Popper; a saber, el de un ideal regulativo. Debido a lo cual, los árbitros frente a la dificultad de encontrar en todos los casos un punto perfecto de distribución eficiente; se hallan obligados a optar por una decisión que contenga la variante de distribución con el mayor grado de correspondencia con el ideal de eficiencia absoluta.

En igual sentido, la corrección de las razones auxiliares dependerá del nivel de aproximación o distancia que tengan aquellas con la razón operativa. De ahí que, en analogía a lo que Popper señala no es completamente necesario -o no es siempre posible- que una razón, en especial las auxiliares, cubra integralmente el criterio de eficiencia, puesto que, para estimarla razonablemente correcta, solo hace falta que alcancen a dicho criterio con una gradualidad importante. Al respecto, si se toma como parámetro de estudio la Figura 4, el proyecto de decisión $x$, y las razones que la soportan, se debe preferir sobre los proyectos de decisión $z$ y $y$, en la medida de que el primero conserva una mayor aproximación al ideal resolutivo c -grado $45^{\circ}$-.

Para explicar esta idea, si configuramos como hipótesis de estudio, que un tribunal arbitral debe laudar en equidad, una controversia donde las partes celebraron un contrato de arrendamiento de un vehículo para uso no comercial, en el cual el arrendatario se obligó a devolver el vehículo materia del contrato en el mismo estado que tenía cuando se le fue entregado; devolviendo posteriormente con un daño que compromete el $20 \%$ de la funcionalidad del vehículo. Sin embargo, también se conoce que el vehículo gozaba de un seguro contra daños que cubría hasta un $15 \%$ de deterioros - 
sin importar la causa de estos-. Los árbitros como se dijo antes, al estar constreñidos a buscar un reparto eficiente de las cargas e intereses económicos de las partes, de forma tal que no sea posible realizar una modificación en tal distribución sin que se traduzca en un empeoramiento de una de las partes; deberán preferir una decisión en la cual se le ordene al demandado el pago de un valor equivalente a la parte que no se hallaba cubierta por el contrato de aseguramiento, es decir el 5\% excedente al porcentaje protegido por la prima. En este sentido, con esta decisión se tutela de forma equitativa los intereses económicos de ambas partes, en fin, se manda que se le pague al actor aquel monto de dinero en el cual no hubiese incurrido en su status quo ante, en fin, el costo de la prima habría sido un gasto que el arrendador hubiese asumido así no se hubiese presentado el siniestro del vehículo. Esta solución hipotética no habría podido operarse en un ejercicio de resolución stricti iuris, por cuanto en dicho caso la lectura de la controversia hubiera sido netamente jurídica, y se hubiera compelido al demandado al pago total del daño efectuado. Sin embargo, como se dijo antes, no siempre es posible alcanzar una solución tan matemática como el de la hipótesis, lo importante es que la decisión logre el mayor grado de aproximación con el ideal resolutivo $C$.

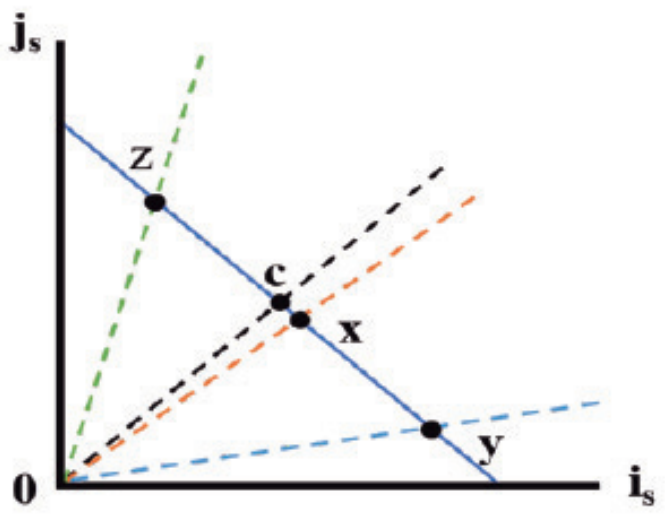

Figura 4 


\subsubsection{La decisión en Equidad, una forma de regulación ex post de los costos de transacción}

Pero, como quizá se pudo haber advertido, por sí solo el concepto de eficiencia de Pareto no construye una razón operativa lo suficientemente coherente para la orientación del arbitraje en equidad, en fin, el punto C, tal y cual se nos ha presentado, marca en exclusiva un punto de distribución neutral e impersonal, lo cual no necesariamente conlleva a afirmar la justicia de una decisión, sino tan solo, su equilibrio económico. Ya autores como Liborio Hierro han sostenido ideas semejantes, cuando han declarado que este criterio “es tan neutral, en efecto, que impide cualquier decisión interesante desde el punto de vista normativo ${ }^{41}$.

Es más, el cumplimiento estricto y no personalizado del esquema referido, puede conducir a cometer sendos errores que lindarían con lo ilógico. Por ejemplo, si se recrea un litigio hipotético donde los árbitros deben determinar el monto indemnizatorio para el resarcimiento de un incumplimiento contractual, dentro del cual el actor solicita el pago total de lo adeudado y el demandado se excepciona negándolo completamente; no aparenta equidad ni justicia, el hecho de que los árbitros, sin detenerse a revisar el contexto circundante, estén obligados a optar por la decisión del punto $C$, la cual en el presente caso equivaldría a condenar al demandado solamente al pago de la mitad de lo que adeuda, ya que en fin, en dicho monto se alcanzaría un punto de equilibrio entre el incumplimiento, como ausencia total de pago, y el cumplimiento como solución efectiva de la obligación. Demostrando la poca efectividad del óptimo de Pareto para ciertos casos; en efecto, tal como se mencionó antes, la eficiencia de Pareto actúa de mejor manera cuando existen dos intereses económicos que son preferidos a la

41. L. Hierro, “Justicia, igualdad y eficiencia”, Isonomía: Revista de Teoría y Filosofía del Derecho, No. 9, 1998, p. 134. 
vez, y que por tanto pueden expresarse mediante curvas de indiferencia.

Es debido a aquello que nace la necesidad de averiguar otras razones de corte moral que sirvan de complemento al criterio de eficiencia y permitan construir una razón operativa completa para la argumentación de los laudos en equidad. Frente a esto, probablemente la solución para el dilema de las "restricciones igualitarias" que provoca la eficiencia de Pareto, se encuentre más cerca de lo que se habría pensado. Esto si se considera que uno de los mecanismos que mayor fuerza ha tomado dentro de los últimos años, al interior de la microeconomía; a saber, la regulación ex post de los costos de transacción, nos puede ofrecer una oportunidad fuerte para erigir una pretensión de corrección no neutralizante. En virtud de lo expuesto, la institución arbitral pasaría a convertirse en un mecanismo de regulación ex post de las transacciones del mercado, que a diferencia de la regulación estatal no comprende un tipo de intervención pública-externa al medio concurrente, sino que puede apreciarse como un método de auto asepsia originado al interno del propio sector privado de la economía.

En este sentido, COASE en su famoso teorema sostiene que "las economías siempre pueden alcanzar una asignación eficiente, incluso en presencia de externalidades, siempre y cuando los costos de transacción sean suficientemente bajos" ${ }^{42}$. Respecto a esto, la microeconomía descubre que las principales fuentes del incremento de los costos dentro de las transacción comerciales, son un grupo de situaciones circundantes a los propios actos de la transacción, relacionados en su mayoría con problemas de información asimétrica, oportunismo y especificidad de $\operatorname{activos}^{43}$.

42. P. Krugman \& R. Wells, Introducción a la Economía: Microeconomía, Reverté, 2007, p. 470.

43. M. CRuz, Una aproximación a la política de los costes de transacción a través del análisis constitucional comparado, Tesis de Doctorado, Universidad de Valladolid, 2000, p. 27. 
Es así como la regulación o corrección ex post de esta clase de problemas transaccionales, concibe a una razón operativa de mayor fuerza y precisión, para el sostenimiento del ánimo argumentativo de los laudos en equidad; en razón de la cual, la tarea de los árbitros al momento del laudaje equitativo no se convierte en un simple rastreo de un punto de equilibrio general $C$, sino que trasciende el campo de la regulación del mercado, especialmente en lo relativo a la cancelación de beneficios o ventajas económicas obtenidas en escenarios de selección adversa y riesgo moral ${ }^{44}$. En virtud de lo cual, al convertirse la corrección de estos fenómenos económicos, junto a la búsqueda de la eficiencia ${ }^{45}$, en el fin último del arbitraje en equidad, se estaría respondiendo positivamente a la máxima que Coase le confiere al derecho, esto es, la reducción de los costos de transacción, la tutela de la competencia perfecta en los mercados y la clarificación de los derechos de propiedad ${ }^{46}$.

Finalmente, en nombre de todos los argumentos planteados es que, desde la nueva perspectiva planteada inspirada en los criterios de eficiencia económica y el teorema de Coase; el arbitraje en equidad pasa a alcanzar un grado de sistema autónomo, cuyo modelo argumentativo no necesita de razones sistémicas - propio de la operatividad del arbitraje en derecho-; sino que fundamente su razón completa,

44. Por selección adversa se entiende, al problema que surge "cuando una parte de la transacción posee información relevante desconocida por la otra, de forma que la primera realiza el proceso decisional y la transacción en superioridad de condiciones", provocando que la misma se lucre de una situación de fijación unilateral de precios o valor -sea que la relación fuese contractual o no-; por su parte, se habla de riesgo moral "cuando una parte de la transacción puede adoptar determinadas acciones que afectan a la valoración que la otra parte ha hecho de la transacción -una vez que ya ha nacido-, pero que esta no puede controlar perfectamente". J. Gómez, "Información Asimétrica: Selección Adversa y Riesgo Moral", Actualidad Empresarial, No. 170, 2008, p. 171.

45. Es importante observar que en la teoría de Coase, sigue vigente la idea de la relación procesal arbitral como una relación económica transmutada, tan solo que aquella se observa en unidad de acto con la relación económica pre-procesal, es decir, la medición de la satisfacción de los intereses económicos de las partes abarca sólo lo referente a la acción y la contestación, sino que analiza también los beneficios prestacionales.

46. Universidad de Valencia, Análisis Económico del Derecho, 15/02/2019, p. 3, <https://bit. $1 y / 2$ YwNIWY> 
entendida como el "conjunto de premisas no superfluas de un razonamiento práctico válido", en el alcance del equilibrio general de las transacciones y el allanamiento de costos injustificados; cumpliendo esta conjugación de criterios con las cuatros propiedad básicas que Iturralde citando a Nino le reconoce a una razón para calificarla como operativa, siendo ellos: (i) la autonomía, o funcionalidad no dependiente de las circunstancias donde se formuló la razón; (ii) la generalidad, (iii) la supervivencia ${ }^{47}$; y, (iv) el carácter integrador con las otras premisas -auxiliares $-{ }^{48}$.

\section{ConClusión}

La presente investigación ha tenido por finalidad la reconstrucción ideológica o de deber ser de la institución del arbitraje en equidad, a fin de responder a la necesidad imperiosa de autonomía metodológica de la cual ha adolecido la modalidad resolutiva en mención, como consecuencia de la vigencia y amplia acogida en la doctrina de la denominada tesis conservadora, que le otorga a las decisiones ex aequo et bono un carácter residual, a manera de una subforma excepcional de decisión al interior del arbitraje en derecho.

Para el efecto, se ha propuesto una serie de argumentos para abrazar una teoría liberal esta modalidad de arbitramiento, mediante la cual se abandone la clásica dependencia a razones sistémicas y autoritativas, para adoptar un modelo de argumentación practica y material, donde las premisas estén cargadas de un contenido inminentemente moral. Para el presente caso, el modelo prescriptivo ensayado, se ha válido de los criterios económicos de eficiencia y del famoso teorema

47. Para ItURRALDE, la supervivencia es "la propiedad que puede justificar la discriminación entre dos razones a los fines de una razón operativa no solo debe ser genérica, sino que también debe ser fáctica en el sentido de no estar identificada meramente por la aplicabilidad de esta u otra razón operativa". V. ItURRALDE, "Acotaciones sobre el principio de unidad", Anuario de Filosofía del Derecho, 2012, pp. 89-90.

48. Ibídem. 
de Coase, en virtud de los cuales el árbitro o los árbitros que estuviesen conociendo una controversia a resolverse en Equidad, deberán tener como máximas de actuación la consecución de un punto de equilibrio distributivo de los intereses económicos de las partes -ahorro y lucro-; así como, la depuración de aquellos "costos de transacción" de los cuales alguna de las partes se hubiese aprovecha de manera injustificada, como producto de los fallos del mercado y una situación de asimetría informática.

No obstante, tal como se dijo en el cuerpo de esta investigación, la corrección de las decisiones en equidad, si bien se medirá según su correspondencia con los criterios económicos señalados, tal correspondencia no será necesariamente cabal, sino que deberá ostentar un carácter gradual; de forma tal, que los parámetros económico-morales de eficiencia establecidos funcionen como un ideal regulativo y no como las premisas mayores del modelo típico de razonamiento judicial -silogístico-.

Empero todos los beneficios que se desprenden del modelo prescriptivo expuestos no alcanzarán el impacto real deseable, y por tanto no superarán el ámbito de la discusión doctrinal, si es que la propia legislación no decide blindar al arbitraje en Equidad, con mecanismos de protección y de depuración argumentativa, como lo sería una causal de nulidad de laudo por la adopción de los esquemas strictis iuris en arbitrajes ex aequo et bono. Mientras esto no suceda, los árbitros seguirán desacatando la voluntad de las partes de someterse a una resolución equitativa -expresa o tácita-, prefiriendo resolver dichas situaciones como si de arbitraje en derecho se tratará; lo que en instancia última se interpone en el camino para alcanzar un sistema jurisdiccional más eficiente y tuitivo. 